\title{
Semianalytic Solution of Space-Time Fractional Diffusion Equation
}

\author{
A. Elsaid, S. Shamseldeen, and S. Madkour \\ Mathematics \& Engineering Physics Department, Faculty of Engineering, Mansoura University, Mansoura 35516, Egypt \\ Correspondence should be addressed to S. Shamseldeen; samir@mans.edu.eg
}

Received 30 April 2016; Accepted 3 July 2016

Academic Editor: Elena Kaikina

Copyright (C) 2016 A. Elsaid et al. This is an open access article distributed under the Creative Commons Attribution License, which permits unrestricted use, distribution, and reproduction in any medium, provided the original work is properly cited.

We study the space-time fractional diffusion equation with spatial Riesz-Feller fractional derivative and Caputo fractional time derivative. The continuation of the solution of this fractional equation to the solution of the corresponding integer order equation is proved. The series solution of this problem is obtained via the optimal homotopy analysis method (OHAM). Numerical simulations are presented to validate the method and to show the effect of changing the fractional derivative parameters on the solution behavior.

\section{Introduction}

Fractional derivatives have been utilized for describing numerous models in different branches of applied science. Anomalous diffusion is one process that has been successfully modeled by fractional derivatives. This type of diffusion is characterized by the nonlinear dependence of the mean square displacement $x(t)$ of a diffusing particle over time $t$ : $x^{2}(t) \propto k_{\alpha} t^{\alpha}$, and it can be interpreted as the Lévy stable densities. On the other hand, in the case of classical diffusion, linear dependence $x^{2}(t) \propto k t$ occurs and it follows Gaussian statistics and Fick's second law for running processes at time $t[1]$.

Anomalous diffusion is described by fractional partial differential equations (FPDEs) by replacing classical derivatives with derivatives of fractional order. A type of anomalous diffusion that received great attention is the fractional diffusion equation with spatial Riesz and Riesz-Feller fractional derivatives $[2,3]$. These fractional derivative operators are used to model phenomena as anomalous diffusion [1], discrete random walk models [3, 4], and continuous random walk models [5] in the form of FPDEs.

General solutions are obtained for some classes of linear FPDEs, using integral transforms. For example, in [6], a general solution is given for a time fractional diffusion-wave equation defined in a bounded space domain. The fractional time derivative is described in the Caputo sense. The finite sine Laplace transform technique is used to obtain the solution. In [7], analytic techniques are introduced to solve certain types of multiterm time-space Caputo-Riesz fractional advection-diffusion equations on a finite domain using the equivalent relationship between the Laplacian operator and the Riesz fractional derivative. In [8], a fundamental solution is investigated for the space-time fractional diffusion-wave equation, which is obtained from the standard diffusion equation by replacing the second-order space derivative with a Riesz-Feller derivative and the first-order time derivative with a Caputo derivative. The paper [9] presents a solution of a unified reaction-diffusion equation with the RiemannLiouville fractional derivative as the time derivative and Riesz-Feller derivative as the space derivative in terms of $H$-function. In [10], a closed form solution in terms of Mittag-Leffler function is obtained for a space-time fractional telegraph equation with Riesz-Feller derivative as the space derivative. In [11], a solution of a space-time fractional advection-dispersion equation is derived in terms of the Green functions.

The proposed abovementioned methods work in case of linear FPDEs. However, for the nonlinear case, the numerical methods and the semianalytic approaches have the advantage. Several numerical techniques are introduced in the literature, and we state some of the known methods here. In [12], 
the matrix transform method with the method of lines is used to obtain the numerical solution of a fractional partial differential equation with Riesz space fractional derivatives on a finite domain. An explicit finite-difference approximation is used to solve the variable-order nonlinear space fractional diffusion equation [13]. Crank-Nicolson method for the fractional diffusion equation with the Riesz fractional derivative is used in [14], while the improved matrix transform method with Pade approximation is used in [15]. Yet, semianalytic solutions for this type of problems include only the series solution by the variational iteration method approach (see $[16,17])$ and by the homotopy analysis method (HAM) (see, e.g., $[2,18])$. In [19], the homotopy analysis method with the Fourier transform is used to obtain and analytically approximate solutions of Riesz fractional diffusion equation and Riesz fractional advection-dispersion equation.

As seen in the literature review, only few articles dealt with applying iterative techniques to these types of FPDEs due to the difficulty of repeated application of Riesz or RieszFeller fractional derivatives to the solution components. In this work, we consider the space-time fractional diffusion equation of the following form:

$$
\begin{aligned}
{ }^{C} D_{t}^{\beta} u(x, t) & =k(u) R_{x}^{\alpha, \theta} u(x, t)+g(u), \\
& -\infty<x<\infty, t>0, \\
u(x, 0) & =f(x),
\end{aligned}
$$

where ${ }^{C} D_{t}^{\beta}$ denotes the Caputo fractional derivative (in time) of order $\beta$ and $R_{x}^{\alpha, \theta}$ denotes the Riesz-Feller fractional derivative (in space) of order $\alpha$ and skewness $\theta$. Parameters $\alpha, \theta$, and $\beta$ are real numbers restricted to

$$
\begin{aligned}
0 & <\alpha \leq 2, \quad \alpha \neq 1, \\
|\theta| & \leq \min (\alpha, 2-\alpha), \\
0 & <\beta \leq 1,
\end{aligned}
$$

and the two functions $k$ and $g$ are continuous functions in $u$. We aim to establish the continuation of the solution of (1) to the exact solution of the corresponding equation in Riesz fractional derivative as the skewness parameter approaches zero. This objective is carried out theoretically then via approximate series solution obtained iteratively by applying the OHAM.

This paper is organized as follows. In Section 2, basic definitions of fractional derivative operators involved are presented. Proof of solution continuation is presented in Section 3. The OHAM is illustrated in Section 4. The results of numerical experiments are presented in Section 5. Section 6 contains the conclusion of this work.

\section{Fractional Derivatives and Integrals}

Definition 1. A real function $f(x), x>0$, is said to be in the space $C_{\mu}, \mu \in \mathbb{R}$, if there exists a real number $p>\mu$, such that $f(x)=x^{p} f_{1}(x)$, where $f_{1}(x) \in C(0, \infty)$, and it is said to be in the space $C_{\mu}^{m}$ if $f^{m} \in C_{\mu}, m \in \mathbb{N}$.
Definition 2. The Riemann-Liouville fractional integral operator of order $\alpha \geq 0$ of a function $f(x) \in C_{\mu}, \mu \geq-1$, is defined as

$$
J^{\alpha} f(x)=\frac{1}{\Gamma(\alpha)} \int_{0}^{x}(x-\tau)^{\alpha-1} f(\tau) d \tau,
$$

$$
\alpha>0, x>0,
$$

$J^{0} f(x)=f(x)$

The operator $J^{\alpha}$ satisfies the following properties. For $f \in$ $C_{\mu}, \mu \geq-1, \alpha, \beta \geq 0$, and $\gamma>-1$,

(1) $J^{\alpha} J^{\beta} f(x)=J^{\alpha+\beta} f(x)$,

(2) $J^{\alpha} J^{\beta} f(x)=J^{\beta} J^{\alpha} f(x)$,

(3) $J^{\alpha} x^{\gamma}=(\Gamma(\gamma+1) / \Gamma(\gamma+\alpha+1)) x^{\alpha+\gamma}$.

Definition 3. The fractional derivative in Caputo sense of $f(x) \in C_{-1}^{m}, m \in \mathbb{N}, x>0$, is defined as

$$
{ }^{C} D_{x}^{\beta} f(x)= \begin{cases}J^{m-\beta} \frac{d^{m}}{d x^{m}} f(x), & m-1<\beta<m, \\ \frac{d^{m}}{d x^{m}} f(x), & \beta=m .\end{cases}
$$

Some basic properties of Caputo fractional derivative are listed in the following lemma.

Lemma 4. If $m-1<\alpha \leq m, m \in \mathbb{N}$, and $f \in C_{\mu}^{m}, \mu \geq-1$, then,

$$
\begin{aligned}
& D^{\alpha}(c)=0, \quad(c \text { is a constant }) \\
& D^{\alpha} x^{\gamma}= \begin{cases}\frac{\Gamma(\gamma+1)}{\Gamma(\gamma-\alpha+1)} x^{\alpha+\gamma}, & \gamma>\alpha-1, \\
0, & \gamma \leq \alpha-1,\end{cases}
\end{aligned}
$$

$$
\begin{aligned}
& D^{\alpha} J^{\alpha} f(x)=f(x), \\
& J^{\alpha} D^{\alpha} f(x)=f(x)-\sum_{k=0}^{m-1} f^{(k)}(0) \frac{x^{k}}{k !}, \quad x>0 .
\end{aligned}
$$

Definition 5. The Riesz-Feller partial fractional derivative $R_{x}^{\alpha, \theta}$ is defined as [3]

$$
\begin{aligned}
R_{x}^{\alpha, \theta} u(x)=-\left[c_{+} D_{+}^{\alpha} u(x)+c_{-} D_{-}^{\alpha} u(x)\right] & \\
& 0<\alpha<2, \alpha \neq 1,
\end{aligned}
$$

where $D_{ \pm}^{\alpha} u(x)$ are the Weyl fractional derivatives defined as

$$
D_{ \pm}^{\alpha} u(x)= \begin{cases} \pm \frac{d}{d x} W_{ \pm}^{1-\alpha} u(x), & 0<\alpha<1 \\ \frac{d^{2}}{d x^{2}} W_{ \pm}^{2-\alpha} u(x), & 1<\alpha<2,\end{cases}
$$


and $W_{ \pm}^{\beta}$ denote the Weyl fractional integrals of order $\beta>0$, given by

$$
\begin{aligned}
& W_{+}^{\beta} u(x)=\frac{1}{\Gamma(\beta)} \int_{-\infty}^{x}(x-z)^{\beta-1} u(z) d z, \\
& W_{-}^{\beta} u(x)=\frac{1}{\Gamma(\beta)} \int_{x}^{\infty}(z-x)^{\beta-1} u(z) d z .
\end{aligned}
$$

The coefficients $c_{+}$and $c_{-}$have the following forms:

$$
c_{ \pm}=\frac{\sin ((\alpha \mp \theta) \pi / 2)}{\sin (\alpha \pi)}
$$

where $|\theta| \leq \min (\alpha, 2-\alpha)$. When $\alpha=0$, the Weyl fractional derivative degenerates into the identity operator:

$$
D_{ \pm}^{0} u(x)=I u(x)=u(x) .
$$

For continuity, we get

$$
\begin{aligned}
& D_{ \pm}^{1} u(x)= \pm \frac{d}{d x} u(x), \\
& D_{ \pm}^{2} u(x)=\frac{d^{2}}{d x^{2}} u(x) .
\end{aligned}
$$

Evidently, in the case of $\alpha=2$ and $\theta=0$, we define

$$
R_{x}^{\alpha, 0} u(x)=\frac{d^{2}}{d x^{2}} u(x) .
$$

For the case of $\alpha=1$ and $\theta=0$, we have

$$
R_{x}^{1,0} u(x)=\frac{d}{d x} H u(x)=\frac{d}{d x} \frac{1}{\pi} \int_{-\infty}^{\infty} \frac{u(z)}{z-x} d z,
$$

where $H$ is the Hilbert transform and the integral is understood in the Cauchy principal value sense. For the cases $\alpha=1$ and $\theta= \pm 1$, we obtain the first derivative:

$$
R_{x}^{1, \pm 1} u(x)= \pm \frac{d}{d x} u(x) .
$$

The special case $\theta=0$ is known as Riesz fractional derivative.

\section{Optimal Homotopy Analysis Method (OHAM)}

In this section, we begin by illustrating the basic steps of the classical homotopy analysis method (HAM). Consider the following nonlinear equation:

$$
N[u(x, t)]=0,
$$

where $N$ is a nonlinear operator, $u(x, t)$ is the unknown function, and $x$ and $t$ denote spatial and temporal independent variables, respectively. Liao [20] generalized the traditional homotopy method to construct the so-called zeroorder deformation equation given by

$$
\begin{gathered}
(1-p) L\left[\phi(x, t ; p)-u_{0}(x, t)\right] \\
=p \hbar H(x, t) N[\phi(x, t ; p)],
\end{gathered}
$$

where $p \in[0,1]$ is an embedding parameter, $\hbar$ is a nonzero auxiliary parameter, $H(x, t)$ is an auxiliary function, $L$ is an auxiliary linear operator, $u_{0}(x, t)$ is an initial guess of $u(x, t)$, and $\phi(x, t ; p)$ is an unknown function. Obviously, when $p=0$ and $p=1$, we have $\phi(x, t ; 0)=u_{0}(x, t)$ and $\phi(x, t ; 1)=u(x, t)$, respectively. Thus, as $p$ increases from 0 to 1 , the solution $\phi(x, t ; p)$ varies from the initial guess $u_{0}(x, t)$ to the required solution $u(x, t)$. By expanding $\phi(x, t ; p)$ in Taylor series with respect to $p$, we have

$$
\phi(x, t ; p)=u_{0}(x, t)+\sum_{m=1}^{\infty} u_{m}(x, t) p^{m},
$$

where

$$
u_{m}(x, t)=\left.\frac{1}{m !} \frac{\partial^{m} \phi(x, t ; p)}{\partial p^{m}}\right|_{p=0} .
$$

If the auxiliary linear operator, the initial guess, and the auxiliary parameter $\hbar$ and the auxiliary function are so properly chosen, then, as proved by Liao [20], series (17) converges at $p=1$ and one has

$$
u(x, t)=u_{0}(x, t)+\sum_{m=1}^{\infty} u_{m}(x, t),
$$

which, as proven by Liao [20], must be one of the solutions of the original nonlinear equation. Using definition (18), the governing equation of the HAM can be deduced from the zero-order deformation equation (16) as follows. Define the vector

$$
\vec{u}_{n}=\left\{u_{0}(x, t), u_{1}(x, t), u_{2}(x, t), \ldots, u_{n}(x, t)\right\} .
$$

From (16), the so-called $m$ th-order deformation equation is given by

$$
\begin{aligned}
& L\left[u_{m}(x, t)-\chi_{m} u_{m-1}(x, t)\right] \\
& =\hbar H(x, t) \mathfrak{R}_{m}\left[\vec{u}_{m-1}(x, t)\right],
\end{aligned}
$$

where

$$
\begin{aligned}
\mathfrak{R}_{m}\left[\vec{u}_{m-1}\right] & =\left.\frac{1}{(m-1) !} \frac{\partial^{m-1} N[\phi(x, t ; p)]}{\partial p^{m-1}}\right|_{p=0}, \\
\chi_{m} & = \begin{cases}0, & m \leq 1, \\
1, & m>1 .\end{cases}
\end{aligned}
$$

Applying the inverse operator $L^{-1}$ to both sides of (21), $u_{m}(x, t)$ can be calculated by symbolic computations software. The HAM has been successfully applied to solve various classes of equations and applied problems [21-25].

In the classical HAM, choosing the value of parameter $\hbar$ depends on inspecting the graph of the quantity of interest: the solution or one of its derivatives. Yet, when $H(x, t)$ is fixed, it is obvious that $u_{m}(x, t)$ contains only one control parameter $\hbar$. Thus, by constructing a formula for the residual error, the OHAM solution is obtained by choosing the value 
for parameter $\hbar$ that minimizes the error. Here, the averaged residual error defined for ordinary differential equations in [26] is generalized to the case of two variable partial differential equations in the following form:

$$
E_{m}(\hbar)=\frac{1}{M K} \sum_{i=0}^{M} \sum_{j=0}^{K}\left[N\left(\sum_{n=0}^{m} u_{n}\left(\frac{i}{M}, \frac{j}{K}\right)\right)\right]^{2},
$$

which is a nonlinear algebraic equation of one unknown: the convergence-control parameter $\hbar$. Thus, the optimal value of $\hbar$ is determined by the minimum of the averaged residual error $E_{m}$ to ensure the fast convergence of the homotopy series.

\section{Continuation of the Solution}

In this section, we define Riesz-Feller fractional derivative on unbounded domain in Caputo sense. This definition is considered as a generalization of the definition given in [27] for Riesz fractional derivative on bounded domains.

Definition 6. The Riesz-Feller fractional derivative in Caputo sense is defined by

$$
\begin{aligned}
{ }^{C} R_{t}^{\alpha, \theta} f(t)=-\left[\left(c_{+}\right){ }^{C} D_{+}^{\alpha}+\left(c_{-}\right){ }^{C} D_{-}^{\alpha}\right] & \\
& 0<\alpha<2, \alpha \neq 1,
\end{aligned}
$$

where ${ }^{C} D_{+}^{\alpha}$ and ${ }^{C} D_{-}^{\alpha}$ are Weyl fractional derivatives defined in Caputo sense for $\alpha \in(0,2), \alpha \neq 1$, as

$$
{ }^{C} D_{ \pm}^{\alpha} f(t)= \begin{cases} \pm W_{ \pm}^{1-\alpha} \frac{d}{d t} f(t), & 0<\alpha<1, \\ W_{ \pm}^{2-\alpha} \frac{d^{2}}{d t^{2}} f(t), & 1<\alpha<2,\end{cases}
$$

and defined for $\alpha=0,1$, and 2 as

$$
\begin{aligned}
& { }^{C} D_{ \pm}^{0} f(t)=f(t), \\
& { }^{C} D_{ \pm}^{1} f(t)= \pm \frac{d}{d t} f(t), \\
& { }^{C} D_{ \pm}^{2} f(t)=\frac{d^{2}}{d t^{2}} f(t) .
\end{aligned}
$$

Lemma 7. Let $f(x)$ belong to the class of "good functions" [28]. Then, for $\alpha \in(0,2), \alpha \neq 1$,

$$
{ }^{C} R_{x}^{\alpha, \theta} f(x)=R_{x}^{\alpha, \theta} f(x) .
$$

Proof. Consider the case $\alpha \in(0,1)$. Riesz-Feller fractional derivative can be written in the following form:

$$
R_{x}^{\alpha, \theta} f(x)=\frac{-d}{d x}\left[\int_{0}^{\infty} \frac{c_{+} f(x-\lambda)-c_{-} f(x+\lambda)}{\lambda^{\alpha}} d \lambda\right],
$$

which can be written as

$$
R_{x}^{\alpha, \theta} f(x)=\int_{0}^{\infty} \frac{c_{-} f^{\prime}(x+\lambda)-c_{+} f^{\prime}(x-\lambda)}{\lambda^{\alpha}} d \lambda .
$$

For ${ }^{C} R_{x}^{\alpha, \theta}$, we have

$$
\begin{aligned}
{ }^{C} R_{x}^{\alpha, \theta} f(x)= & c_{+} \int_{-\infty}^{x} \frac{f^{\prime}(z)}{(x-z)^{\alpha}} d z \\
& -c_{-} \int_{x}^{\infty} \frac{f^{\prime}(y)}{(y-x)^{\alpha}} d y
\end{aligned}
$$

which by substitution yields

$$
{ }^{C} R_{x}^{\alpha, \theta} f(x)=\int_{0}^{\infty} \frac{c_{-} f^{\prime}(x+\lambda)-c_{+} f^{\prime}(x-\lambda)}{\lambda^{\alpha}} d \lambda,
$$

which is the same as (29). A similar argument holds for the case $\alpha \in(1,2)$.

As the equivalence between the Riemann and Caputo definitions of Riesz-Feller fractional derivative is deduced, we discuss the continuation of the solution obtained.

Theorem 8. If $f(x)$ is a function in $L^{1}(-\infty, \infty)$, then the exact solution $u_{\beta}^{\alpha, \theta}$ of the space-time fractional diffusion equation (1) is given by

$$
\begin{aligned}
& u_{\beta}^{\alpha, \theta}(x, t) \\
& \quad=\frac{1}{2 \pi} \iint_{-\infty}^{\infty} E_{\beta}\left(-|\omega|^{\alpha} t^{\beta} e^{-i \theta \pi / 2}\right) f(v) e^{i \omega(x-v)} d \omega d v
\end{aligned}
$$

where $E_{\beta}(z)$ is the one parameter Mittag-Leffler function defined as [29]

$$
E_{\beta}(z)=\sum_{k=0}^{\infty} \frac{z^{k}}{\Gamma(1+k \beta)}, \quad \beta>0
$$

Theorem 9. Let $\alpha \in(1,2), \beta \in(0,1),|\theta| \leq \min (\alpha, 2-\alpha)$, and $f(x)$ be a function in $L^{1}(-\infty, \infty)$, and let $u_{\beta}^{\alpha, \theta}$ be solution (32) of the space-time fractional problem (1); then,

$$
\lim _{\theta \rightarrow 0} u_{\beta}^{\alpha, \theta}(x, t)=u_{\beta}^{\alpha}(x, t)
$$

where $u_{\beta}^{\alpha}(x, t)$ is the exact solution of Riesz fractional diffusion equation:

$$
\begin{aligned}
{ }^{C} D_{t}^{\beta} u(x, t) & =R_{x}^{\alpha} u(x, t), \quad-\infty<x<\infty, t>0, \\
u(x, 0) & =f(x),
\end{aligned}
$$

with the same limits for parameters $\alpha$ and $\beta$.

Proof. Consider the set of functions:

$$
\begin{aligned}
& \varphi_{n}(\omega) \\
& =\frac{1}{2 \pi} E_{\beta}\left(-|\omega|^{\alpha} t^{\beta} e^{-i \pi / 2 n}\right) \int_{-\infty}^{\infty} f(v) e^{i \omega(x-v)} d v, \\
& \omega \in(-\infty, \infty), n=1,2, \ldots
\end{aligned}
$$


Then,

$$
\left|\varphi_{n}(\omega)\right| \leq \frac{1}{2 \pi}\left|E_{\beta}\left(-|\omega|^{\alpha} t^{\beta} e^{-i \pi / 2 n}\right)\right| \int_{-\infty}^{\infty}|f(v)| d v,
$$

and as $f(x) \in L^{1}(-\infty, \infty)$, then there exists $M>0$ such that

$$
\left|\varphi_{n}(\omega)\right| \leq \frac{M}{2 \pi}\left|E_{\beta}\left(-|\omega|^{\alpha} t^{\beta} e^{-i \pi / 2 n}\right)\right| .
$$

From [30, Theorem (1.6)], there exists $K_{1}>0$ such that

$$
\left|E_{\beta}(-z)\right| \leq \frac{K_{1}}{1+|z|} \text {. }
$$

Then,

$$
\begin{aligned}
\left|\varphi_{n}(\omega)\right| \leq \frac{1}{2 \pi} \frac{M K_{1}}{1+\left.|| \omega\right|^{\alpha} t^{\beta \mid}}, & \\
& \omega \in(-\infty, \infty), n=1,2, \ldots .
\end{aligned}
$$

By Lebesgue dominated convergence theorem and setting $\theta=$ $1 / n$, we have

$$
\begin{aligned}
\lim _{\theta \rightarrow 0} & u_{\beta}^{\alpha, \theta}(x, t)=\lim _{n \rightarrow \infty} \int_{-\infty}^{\infty} \varphi_{n}(\omega) d \omega \\
& =\int_{-\infty}^{\infty} \lim _{n \rightarrow \infty} \varphi_{n}(\omega) d \omega \\
& =\frac{1}{2 \pi} \iint_{-\infty}^{\infty} E_{\beta}\left(-|\omega|^{\alpha} t^{\beta}\right) f(v) e^{i \omega(x-v)} d \omega d v \\
& =u_{\beta}^{\alpha}(x, t),
\end{aligned}
$$

which is the exact solution of the Riesz fractional diffusion equation (35).

Similar arguments hold to prove the continuation of the solution when both parameters $\alpha$ and $\beta$ tend to their integer limits.

\section{Numerical Simulation}

In this section, we consider linear and nonlinear problems to illustrate the efficiency of the OHAM to this type of problems and to illustrate the continuation of the solution we proved in Section 4. Yet, to apply the recursive technique of OHAM to problem (1), a repeated evaluation of Riesz-Feller fractional derivative to solution components is needed. This is accomplished by using a property of Riesz-Feller fractional derivative that is proved in the following lemma.

Lemma 10. Let $\alpha \in(0,2), \alpha \neq 1,|\theta| \leq \min (\alpha, 2-\alpha)$, and $\omega>0$. Then,

$$
R_{x}^{\alpha, \theta}\left(e^{i \omega x}\right)=-\omega^{\alpha} e^{i(\omega x-\theta(\pi / 2))} .
$$

Proof.

Case $\alpha \in(0,1)$. From the definition of Riesz-Feller fractional derivative and by substitution, we can write

$$
R_{x}^{\alpha, \theta}\left(e^{i \omega x}\right)=\frac{-d}{d x}\left[\int_{0}^{\infty} \frac{c_{+} e^{i \omega(x-\tau)}-c_{-} e^{i \omega(x+\tau)}}{\Gamma(1-\alpha) \tau^{\alpha}} d \tau\right],
$$

which reduces to

$$
\begin{aligned}
& R_{x}^{\alpha, \theta}\left(e^{i \omega x}\right) \\
& \quad=\frac{-i \omega}{\Gamma(1-\alpha)} e^{i \omega x}\left(c_{+} \frac{\Gamma(1-\alpha)}{(i \omega)^{1-\alpha}}-c_{-} \frac{\Gamma(1-\alpha)}{(-i \omega)^{1-\alpha}}\right), \\
& R_{x}^{\alpha, \theta}\left(e^{i \omega x}\right)=-\omega^{\alpha} e^{i \omega x}\left(c_{+} i^{\alpha}-c_{-}(-i)^{\alpha}\right) .
\end{aligned}
$$

Substituting for $c_{+}$and $c_{-}$, we have

$$
\begin{aligned}
& R_{x}^{\alpha, \theta}\left(e^{i \omega x}\right)=-\omega^{\alpha} e^{i \omega x} \\
& \quad \cdot \frac{\sin ((\alpha-\theta) \pi / 2) e^{i \pi \alpha / 2}+\sin ((\alpha+\theta) \pi / 2) e^{-i \pi \alpha / 2}}{\sin (\alpha \pi)} .
\end{aligned}
$$

Utilizing Euler form and trigonometric identities, we have

$$
R_{x}^{\alpha, \theta}\left(e^{i \omega x}\right)=-\omega^{\alpha} e^{i(\omega x-\theta(\pi / 2))} .
$$

Case $\alpha \in(1,2)$. Consider

$$
R_{x}^{\alpha, \theta}\left(e^{i \omega x}\right)=\frac{-d^{2}}{d x^{2}} \int_{0}^{\infty} \frac{c_{+} e^{i \omega(x-\tau)}-c_{-} e^{i \omega(x+\tau)}}{\Gamma(2-\alpha) \tau^{\alpha-1}} d \tau,
$$

which reduces to

$$
\begin{aligned}
& R_{x}^{\alpha, \theta}\left(e^{i \omega x}\right) \\
& \quad=\frac{\omega^{2}}{\Gamma(2-\alpha)} e^{i \omega x}\left(c_{+} \frac{\Gamma(2-\alpha)}{(i \omega)^{2-\alpha}}-c_{-} \frac{\Gamma(2-\alpha)}{(-i \omega)^{2-\alpha}}\right) .
\end{aligned}
$$

Carrying out the same procedure, the lemma follows.

Example 11. Consider problem (1) with $k(u)=1, g(u)=a u$, and $f(x)=\epsilon \sin (\pi x / L)$ :

$$
\begin{aligned}
{ }^{C} D_{t}^{\beta} u(x, t) & =R_{x}^{\alpha, \theta} u(x, t)+a u, \\
-\infty<x<\infty, t>0, & \\
u(x, 0) & =\epsilon \sin \left(\frac{\pi x}{L}\right),
\end{aligned}
$$

where $a, \epsilon$, and $b$ are real constants.

To solve this problem by means of OHAM, let us consider the linear operator

$$
L[\phi(x, t ; p)]={ }^{C} D_{t}^{\beta} \phi(x, t ; p)
$$

and the operator

$$
N[\phi(x, t ; p)]={ }^{C} D_{t}^{\beta} \phi-R_{x}^{\alpha, \theta} \phi-a \phi .
$$

Then, one can write the $m$ th-order deformation equation, with $H(x, t)=1$, for this problem as

$$
L\left[u_{m}(x, t)-\chi_{m} u_{m-1}(x, t)\right]=\hbar \Re_{m}\left[u_{m-1}(x, t)\right],
$$


where

$$
\mathfrak{R}_{m}\left[u_{m-1}\right]={ }^{C} D_{t}^{\beta} u_{m-1}-R_{x}^{\alpha, \theta} u_{m-1}-a u_{m-1} .
$$

Apply the inverse operator $L^{-1}=J_{t}^{\mu}$ to both sides of (52); we obtain

$$
\begin{aligned}
u_{m}(x, t)= & \chi_{m} u_{m-1}(x, t) \\
& +\left(u_{m}(x, 0)-\chi_{m} u_{m-1}(x, 0)\right) \\
& +\hbar J_{t}^{\beta}\left[\Re_{m}\left[u_{m-1}(x, t)\right]\right],
\end{aligned}
$$

where

$$
\begin{aligned}
& u_{0}(x, t)=u(x, 0)=\epsilon \sin \left(\frac{\pi x}{L}\right), \\
& u_{m}(x, 0)=0, \quad m>1 .
\end{aligned}
$$

Referring to (53) and (54) with conditions (55), we get

$$
\begin{aligned}
& u_{1}(x, t)=\frac{h t^{\beta} \epsilon}{\Gamma[1+\beta]}\left(-a \sin \left(\frac{\pi x}{L}\right)+\left(\frac{\pi}{L}\right)^{\alpha} \sin \left(\frac{\pi x}{L}\right.\right. \\
& \left.\left.-\frac{\pi \theta}{2}\right)\right), \\
& u_{2}=\frac{h(1+h) t^{\beta} \epsilon}{\Gamma(1+2 \beta)}\left(-a \sin \left(\frac{\pi x}{L}\right)+\left(\frac{\pi}{L}\right)^{\alpha} \sin \left(\frac{\pi x}{L}\right.\right. \\
& \left.\left.-\frac{\pi \theta}{2}\right)\right)+\frac{h^{2} t^{2 \beta} \epsilon}{\Gamma(1+2 \beta)}\left(\left(a^{2} \sin \left(\frac{\pi x}{L}\right)+\left(\frac{\pi}{L}\right)^{\alpha}\right.\right. \\
& .\left(\left(\frac{\pi}{L}\right)^{\alpha} \sin \left(\frac{\pi x}{L}-\pi \theta\right)\right. \\
& \left.\left.\left.-2 a \sin \left(\frac{\pi x}{L}-\frac{\pi \theta}{2}\right)\right)\right)\right) .
\end{aligned}
$$

The series solution is given by $u=u_{0}+u_{1}+u_{2}+u_{3}+\cdots$. Figure 1 shows the effect of the skewness parameter $\theta$ on the behavior of the solution. The plot represents the sum of the first five terms $\left(u_{0}\right.$ to $\left.u_{4}\right)$ in the OHAM series when $a=1.0, \epsilon=1.0, L=3.0, t=0.2$, and $0<x<\pi$ and the fractional parameters $\beta=1.0, \alpha=1.5$, and $\theta=$ $0,0.5,-0.5$. At these different values of $\theta=0,0.5$, and -0.5 , the optimal convergence-control parameter $\hbar$ is calculated as $\hbar=-0.995787,-1.049435$, and -1.056491 , and the corresponding residual error is obtained as $E=2.969688 *$ $10^{-7}, 4.243949 * 10^{-6}$, and $4.453205 * 10^{-6}$, respectively.

The solution (the first five terms in the OHAM series) behavior as the parameters $\theta, \alpha$, and $\beta$ change at a fixed time $t=5.0$ and in the interval $0 \leq x \leq \pi$ is shown in Figures 1-3. The amplitude of the solution decreases $\alpha$ and/or $\beta$ increases. As $\alpha$ reaches 2 and/or $\beta$ increases up to unity, the series solution approximately coincides with the exact solution of the corresponding integer order equation $u=e^{-\left(\pi^{2} / L^{2}-a\right) t} \sin (\pi x / L)$, represented by the solid line in the graph (refer to Figures 2 and 3 ), and this numerical result supports the continuation of the solution. The series displayed

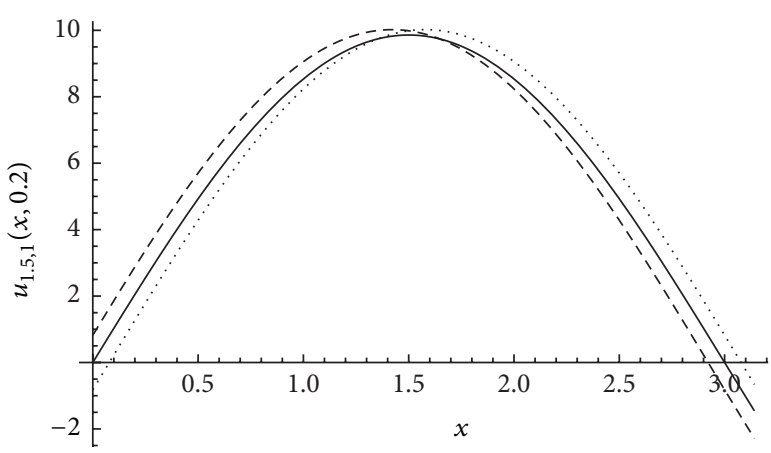

$$
\begin{aligned}
-\theta & =0.0 \\
---\theta & =0.25 \\
\ldots-\theta & =-0.25
\end{aligned}
$$

FIGURE 1: The behavior of the four terms of OHAM solution of (49) at $t=0.2,0<x<\pi$, and the fractional parameters $\alpha=1.5$ and $\beta=1$ and the skewness $\theta=0,0.25,-0.25$.

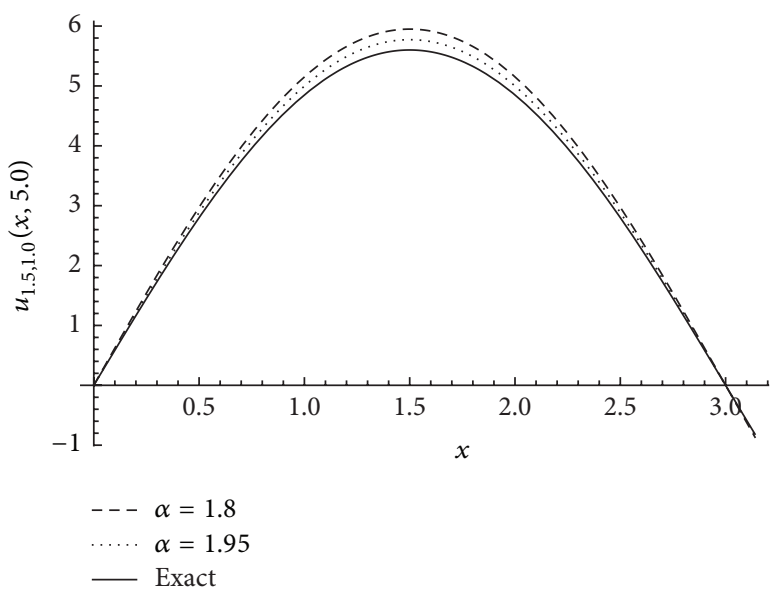

FIGURE 2: The behavior of the four terms of OHAM solution of (49) at $t=0.2,0<x<\pi$, and the fractional parameters $\theta=0, \beta=1$, and $\alpha=1.8,1.95$, compared with the exact solution.

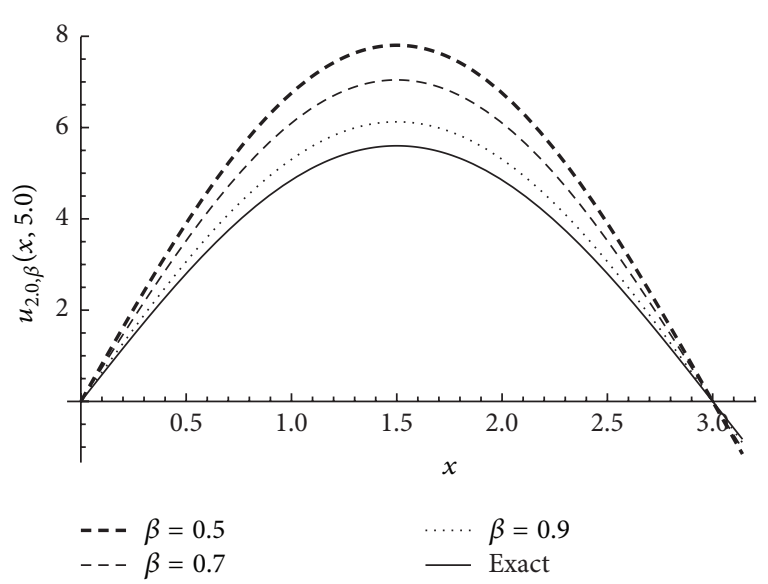

Figure 3: The behavior of the four terms of OHAM solution of (49) at $t=0.2,0<x<\pi$, and the fractional parameters $\theta=0, \alpha=2.0$, and $\beta=0.5,0.7,0.9$, compared with the exact solution. 
TABLE 1: The estimated optimal convergence parameter $\hbar$ and the corresponding residual error $E_{m}$ at different fractional parameters used in Example 11.

\begin{tabular}{|c|c|c|c|}
\hline \multicolumn{2}{|c|}{$\alpha, \theta, \beta$} & Optimal $\hbar$ & Residual error $\left(E_{4}\right)$ \\
\hline \multirow{3}{*}{$\alpha=1.5, \beta=1.0$} & $\theta=0.0$ & -0.990381137 & $4.6032 E-10$ \\
\hline & $\theta=0.25$ & -1.001711905 & $2.5426 E-5$ \\
\hline & $\theta=-0.25$ & -1.0023206958 & $2.54262 E-6$ \\
\hline \multirow{3}{*}{$\beta=1.0, \theta=0$} & $\alpha=1.8$ & -0.9339901124 & $2.2375 E-7$ \\
\hline & $\alpha=1.9$ & -0.9303835167 & $3.4254 E-7$ \\
\hline & $\alpha=1.95$ & -0.9285827510 & $4.2025 E-7$ \\
\hline \multirow{3}{*}{$\alpha=2.0, \theta=0$} & $\beta=0.5$ & -0.9099205063 & $9.7340 E-9$ \\
\hline & $\beta=0.7$ & -0.9151677201 & $7.4716 E-8$ \\
\hline & $\beta=0.9$ & -0.9225579219 & $3.0301 E-8$ \\
\hline
\end{tabular}

in plots is the partial sum of the first five terms: $n=5$ (summing $u_{0}$ to $u_{4}$ ). The optimal convergence parameter $\hbar$ in each case is obtained by minimizing the residual error $E_{m}$ displayed in (23) in the intervals $0 \leq x \leq \pi$ and $0 \leq t \leq 6.0$, and the obtained results are shown in Table 1.

Example 12. Consider the nonlinear fractional problem (1) with $k(u)=u, g(u)=4 u^{2}-u$, and $f(x)=\sin (2 x)$ :

$$
\begin{aligned}
{ }^{C} D_{t}^{\beta} u(x, t) & =u R_{x}^{\alpha, \theta} u(x, t)+4 u^{2}-u, \\
& -\infty<x<\infty, t>0, \\
u(x, 0) & =\sin (2 x) .
\end{aligned}
$$

Here, we choose the auxiliary linear operator as

$$
L[\phi]=\frac{\partial}{\partial t}(\phi),
$$

and the nonlinear operator $N$ is chosen as

$$
N[\phi]={ }^{C} D_{t}^{\beta} \phi-\phi R_{x}^{\alpha, \theta}(\phi)-4 \phi^{2}+\phi
$$

Then, $m$ th-order deformation equation for this problem is given by

$$
\begin{aligned}
\frac{\partial}{\partial t} & {\left[u_{m}(x, t)-\chi_{m} u_{m-1}(x, t)\right] } \\
& =\hbar H(x, t) \Re_{m}\left[\vec{u}_{m-1}(x, t)\right],
\end{aligned}
$$

where $\mathfrak{R}_{m}\left[\vec{u}_{m-1}(x, t)\right]$ is given by

$$
\begin{aligned}
\mathfrak{R}_{m}\left[\vec{u}_{m-1}(x, t)\right]= & { }^{C} D_{t}^{\beta}\left(u_{m-1}\right)-\sum_{k=0}^{m-1} u_{k} R_{x}^{\alpha, \theta}\left(u_{m-1-k}\right) \\
& -4 \sum_{k=0}^{m-1} u_{k} u_{m-1-k}+u_{m-1} .
\end{aligned}
$$

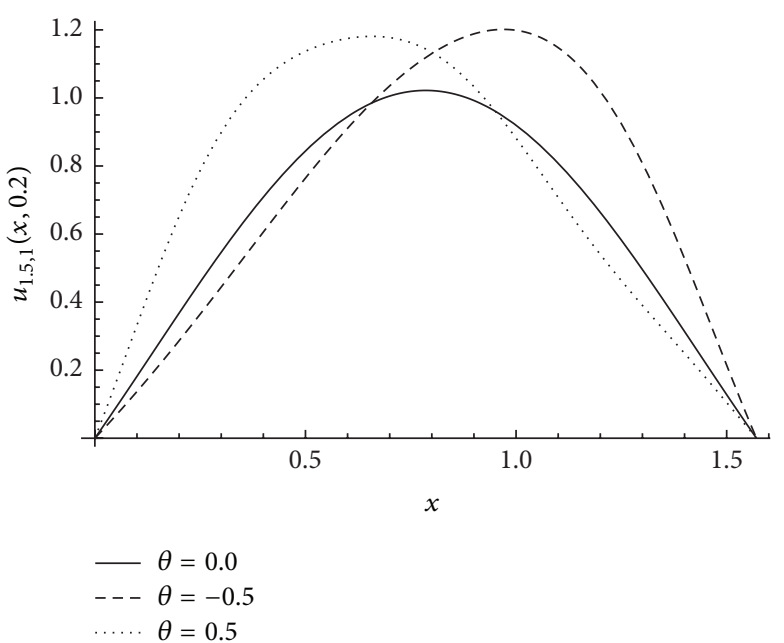

FIGURE 4: The behavior of the four terms of OHAM solution of (57) at $t=0.2,0<x<\pi / 2$, and the fractional parameters $\alpha=1.5$ and $\beta=1$ and the skewness $\theta=0,0.5,-0.5$.

We choose $H(x, t)=1$ and $u_{0}=\sin (2 x)$. Following the same procedure in the previous example to obtain the series solution terms,

$$
\begin{aligned}
u_{0} & =\sin (2 x), \\
u_{1} & =\frac{h t^{\beta}}{2 \Gamma(1+\beta)}\left(-4+4 \cos (4 x)+2^{\alpha} \cos \left(\frac{\pi \theta}{2}\right)\right. \\
& \left.-2^{\alpha} \cos \left(4 x-\frac{\pi \theta}{2}\right)+2 \sin (2 x)\right) .
\end{aligned}
$$

To find the next terms in the solution series, use the following recursive formula which is valid for $m>1$ :

$$
\begin{aligned}
u_{m} & =(1+\hbar) u_{m-1}+\hbar J_{t}^{\beta}\left[-\sum_{k=0}^{m-1} u_{k} R_{x}^{\alpha, \theta}\left(u_{m-1-k}\right)\right. \\
& \left.-4 \sum_{k=0}^{m-1} u_{k} u_{m-1-k}+u_{m-1}\right]
\end{aligned}
$$

and the solution is thus obtained as

$$
u=u_{0}+u_{1}+u_{2}+u_{3}+\cdots .
$$

Figures 4-6 show the solution (the first five terms in the OHAM series) behavior as the parameters $\theta, \alpha$, and $\beta$ change at a fixed time $t=0.2$ and in the interval $0 \leq x \leq$ $\pi / 2$. As $\alpha$ and/or $\beta$ increases, the amplitude of the solution decreases. As $\alpha$ tends to 2 and/or $\beta$ goes up to unity, the series solution approximately coincides with the exact solution of the corresponding integer order equation $u=e^{-t} \sin (2 x)$, represented by the solid line in the graph (see Figures 5 and 6), and this confirms the continuation of the solution numerically. The series displayed in plots is the partial sum of the first five terms: $n=5$ (summing $u_{0}$ to $u_{4}$ ). The optimal convergence parameter $\hbar$ in each case is obtained by minimizing the residual error $E_{m}$ displayed in (23) in the intervals 
TABLE 2: The estimated optimal convergence parameter $\hbar$ and the corresponding residual error $E_{m}$ at different fractional parameters used in Example 12.

\begin{tabular}{|c|c|c|c|}
\hline \multicolumn{2}{|c|}{$\alpha, \theta, \beta$} & Optimal $\hbar$ & Residual error $\left(E_{4}\right)$ \\
\hline \multirow{3}{*}{$\alpha=1.5, \beta=1.0$} & $\theta=0.0$ & -0.831733694 & $2.3498 E-8$ \\
\hline & $\theta=0.5$ & -0.954364814 & $1.0016 E-5$ \\
\hline & $\theta=-0.5$ & -0.637748947 & $7.4733 E-6$ \\
\hline \multirow{3}{*}{$\beta=1.0, \theta=0$} & $\alpha=1.92$ & -0.589337116 & $2.3160 E-6$ \\
\hline & $\alpha=1.95$ & -0.589337116 & $2.1595 E-6$ \\
\hline & $\alpha=1.99$ & -0.639152091 & $7.6926 E-6$ \\
\hline \multirow{3}{*}{$\alpha=2.0, \theta=0$} & $\beta=0.5$ & -0.858637427 & $2.9308 E-9$ \\
\hline & $\beta=0.7$ & -0.929790675 & $7.6259 E-12$ \\
\hline & $\beta=0.95$ & -0.973269800 & $2.7929 E-12$ \\
\hline
\end{tabular}

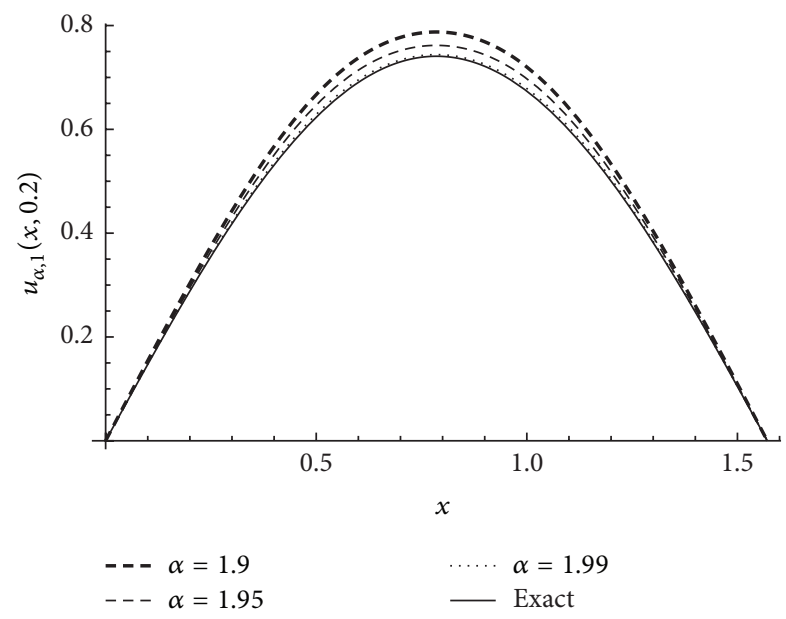

FIgURE 5: The behavior of the four terms of OHAM solution of (57) at $t=0.2,0<x<\pi / 2$, and the fractional parameters $\theta=0, \beta=1$, and $\alpha=1.5,1.7,1.9$, compared with the exact solution.

$0 \leq x \leq 2$ and $0 \leq t \leq 0.4$, and the obtained results are tabulated in Table 2.

\section{Conclusion}

A definition of the fractional-order Riesz-Feller derivative in the Caputo sense is proposed and its equivalence with the classical definition is proved. Then, we proved the continuation of the solution of the fractional-order anomalous diffusion equation with Riesz-Feller spatial derivative to the corresponding Riesz problem. The iterative series solution for the fractional equation is obtained using the OHAM. The advantage of using this technique is the ability to estimate an approximation to the residual error. The results obtained illustrate graphically the continuation of the solution we proved theoretically.

\section{Competing Interests}

The authors declare that there is no conflict of interests regarding the publication of this paper.

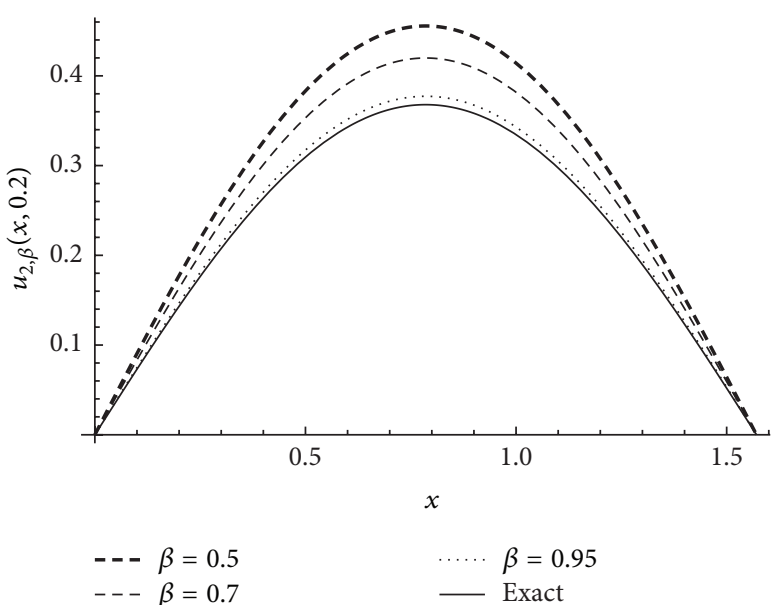

FIGURE 6: The behavior of the four terms of OHAM solution of (57) at $t=0.2,0<x<\pi / 2$, and the fractional parameters $\theta=0, \alpha=2.0$, and $\beta=0.4,0.8,0.95$, compared with the exact solution.

\section{References}

[1] R. Metzler and J. Klafter, “The random walk's guide to anomalous diffusion: a fractional dynamics approach," Physics Reports, vol. 339, no. 1, pp. 1-77, 2000.

[2] A. Elsaid, "Homotopy analysis method for solving a class of fractional partial differential equations," Communications in Nonlinear Science and Numerical Simulation, vol. 16, no. 9, pp. 3655-3664, 2011.

[3] R. Gorenflo, F. Mainardi, D. Moretti, G. Pagnini, and P. Paradisi, "Discrete random walk models for space-time fractional diffusion," Chemical Physics, vol. 284, no. 1-2, pp. 521-541, 2002.

[4] R. Gorenflo and A. Vivoli, "Fully discrete random walks for space-time fractional diffusion equations," Signal Processing, vol. 83, no. 11, pp. 2411-2420, 2003.

[5] R. Gorenflo and F. Mainardi, "Fractional diffusion processes: probability distributions and continuous time random walk," in Processes with Long-Range Correlations, G. Rangarajan and M. Ding, Eds., vol. 621 of Lecture Notes in Physics, pp. 148-166, Springer, Berlin, Germany, 2003.

[6] O. P. Agrawal, "Solution for a fractional diffusion-wave equation defined in a bounded domain," Nonlinear Dynamics, vol. 29, no. 1-4, pp. 145-155, 2002.

[7] H. Jiang, F. Liu, I. Turner, and K. Burrage, "Analytical solutions for the multi-term time-space Caputo-Riesz fractional advection-diffusion equations on a finite domain," Journal of Mathematical Analysis and Applications, vol. 389, no. 2, pp. 11171127, 2012.

[8] F. Mainardi, Y. Luchko, and G. Pagnini, “The fundamental solution of the space-time fractional diffusion equation," Fractional Calculus and Applied Analysis, vol. 4, no. 2, pp. 153-192, 2001.

[9] H. J. Haubold, A. M. Mathai, and R. K. Saxena, "Further solutions of fractional reaction-diffusion equations in terms of the $\mathrm{H}$-function," Journal of Computational and Applied Mathematics, vol. 235, no. 5, pp. 1311-1316, 2011.

[10] M. Garg, A. Sharma, and P. Manohar, "Solution of generalized space-time fractional telegraph equation with composite and riesz-feller fractional derivatives," International Journal of Pure and Applied Mathematics, vol. 83, no. 5, pp. 685-691, 2013. 
[11] F. Huang and F. Liu, "The fundamental solution of the space-time fractional advection-dispersion equation," Journal of Applied Mathematics \& Computing, vol. 18, no. 1-2, pp. 339350, 2005.

[12] Q. Yang, F. Liu, and I. Turner, "Numerical methods for fractional partial differential equations with Riesz space fractional derivatives," Applied Mathematical Modelling, vol. 34, no. 1, pp. 200-218, 2010.

[13] R. Lin, F. Liu, V. Anh, and I. Turner, "Stability and convergence of a new explicit finite-difference approximation for the variable-order nonlinear fractional diffusion equation," Applied Mathematics and Computation, vol. 212, no. 2, pp. 435-445, 2009.

[14] C. Çelik and M. Duman, "Crank-Nicolson method for the fractional diffusion equation with the Riesz fractional derivative," Journal of Computational Physics, vol. 231, no. 4, pp. 1743-1750, 2012.

[15] M. Rahman, A. Mahmood, and M. Younis, "Improved and more feasible numerical methods for Riesz space fractional partial differential equations," Applied Mathematics and Computation, vol. 237, pp. 264-273, 2014.

[16] A. Elsaid, "The variational iteration method for solving Riesz fractional partial differential equations," Computers and Mathematics with Applications, vol. 60, no. 7, pp. 1940-1947, 2010.

[17] V. J. Ervin and J. P. Roop, "Variational solution of fractional advection dispersion equations on bounded domains," Numerical Methods for Partial Differential Equations, vol. 23, no. 2, pp. 256-281, 2007.

[18] X. Zhang, L. Wei, B. Tang, and Y. He, "Homotopy analysis method for space-time fractional differential equations," International Journal of Numerical Methods for Heat \& Fluid Flow, vol. 23, no. 6, Article ID 17092674, pp. 1063-1075, 2013.

[19] S. S. Ray and S. Sahoo, "Analytical approximate solutions of Riesz fractional diffusion equation and Riesz fractional advection-dispersion equation involving nonlocal space fractional derivatives," Mathematical Methods in the Applied Sciences, vol. 38, no. 13, pp. 2840-2849, 2015.

[20] S. Liao, Beyond Perturbation: Introduction to the Homotopy Analysis Method, CRC Press, Boca Raton, Fla, USA, 2003.

[21] S. Liao, "Comparison between the homotopy analysis method and homotopy perturbation method," Applied Mathematics and Computation, vol. 169, no. 2, pp. 1186-1194, 2005.

[22] Y. Bouremel, "Explicit series solution for the Glauert-jet problem by means of the homotopy analysis method," Communications in Nonlinear Science and Numerical Simulation, vol. 12, no. 5, pp. 714-724, 2007.

[23] S. Abbasbandy and F. S. Zakaria, "Soliton solutions for the fifth-order KdV equation with the homotopy analysis method," Nonlinear Dynamics, vol. 51, no. 1-2, pp. 83-87, 2008.

[24] J. Cang, Y. Tan, H. Xu, and S.-J. Liao, "Series solutions of non-linear Riccati differential equations with fractional order," Chaos, Solitons \& Fractals, vol. 40, no. 1, pp. 1-9, 2009.

[25] R. Ellahi, M. Raza, and K. Vafai, "Series solutions of nonNewtonian nanofluids with Reynolds' model and Vogel's model by means of the homotopy analysis method," Mathematical and Computer Modelling, vol. 55, no. 7-8, pp. 1876-1891, 2012.

[26] S. Liao, "An optimal homotopy-analysis approach for strongly nonlinear differential equations," Communications in Nonlinear Science and Numerical Simulation, vol. 15, no. 8, pp. 2003-2016, 2010.
[27] A. El-Sayed and M. Gaber, "On the finite caputo and finite riesz derivatives," Electronic Journal of Theoretical Physics, vol. 3, no. 12, pp. 81-95, 2006.

[28] K. S. Miller and B. Ross, An Introduction to the Fractional Calculus and Fractional Differential Equations, 1993.

[29] I. Podlubny, Fractional Differential Equations, vol. 198 of Methods of Their Solution and Some of Their Applications, Academic Press, 1999.

[30] H. Zhang and F. Liu, "The fundamental solutions of the space, space-time Riesz fractional partial differential equations with periodic conditions," Numerical Mathematics-English Series, vol. 16, no. 2, pp. 181-192, 2007. 


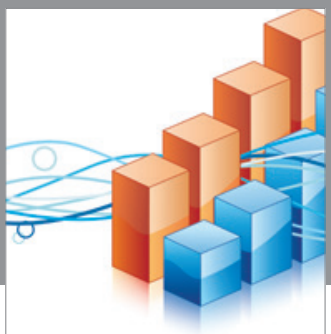

Advances in

Operations Research

vatem alat4

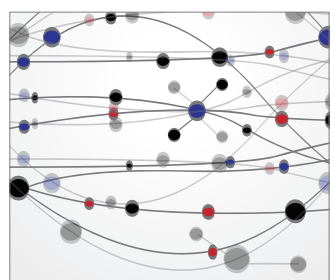

\section{The Scientific} World Journal
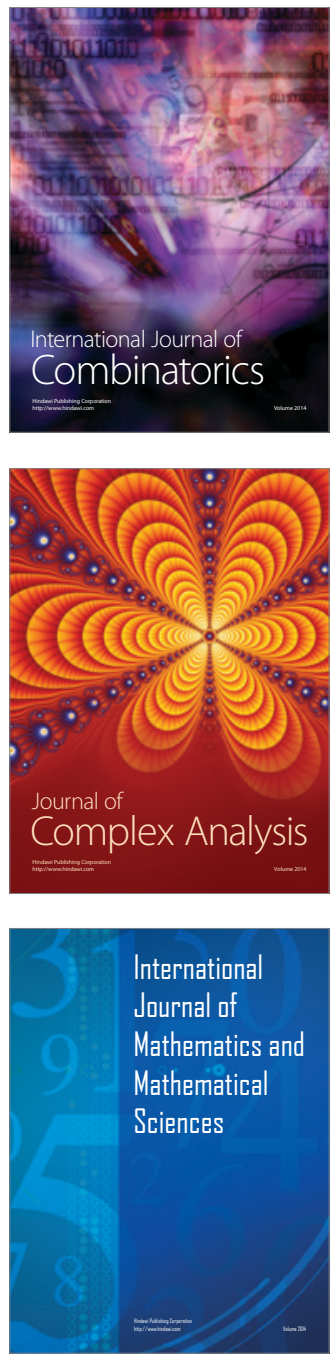
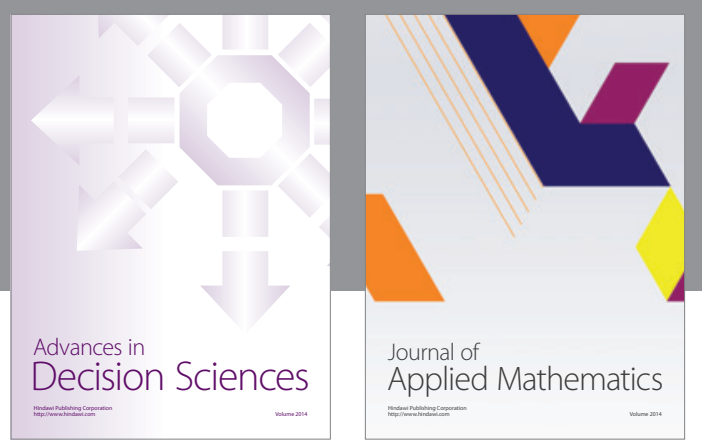

Algebra

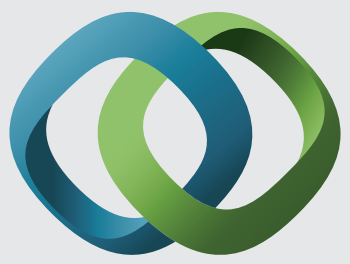

\section{Hindawi}

Submit your manuscripts at

http://www.hindawi.com
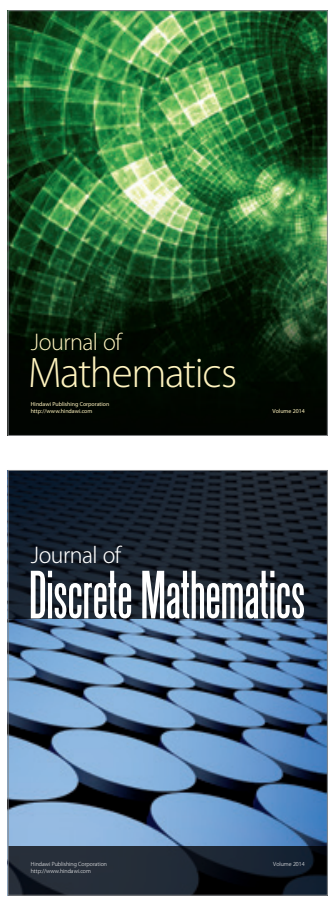

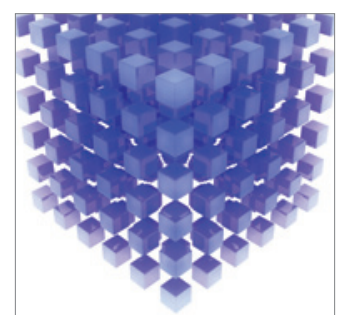

Mathematical Problems in Engineering
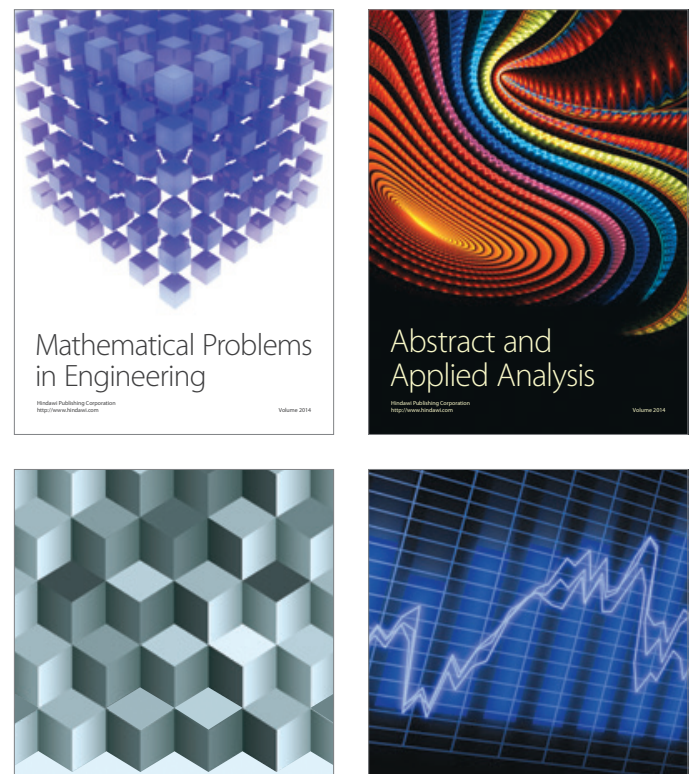

Journal of

Function Spaces

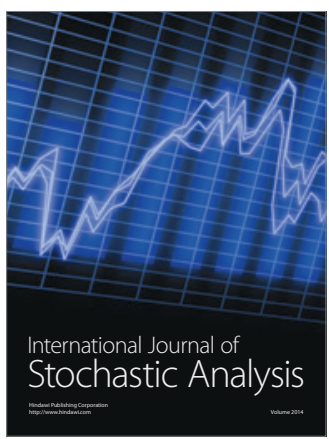

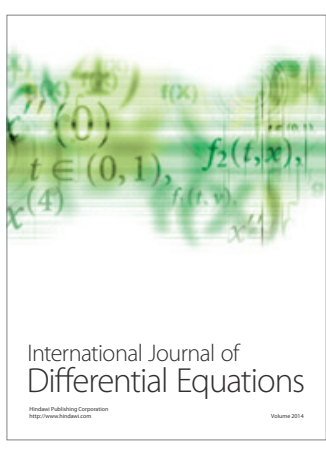
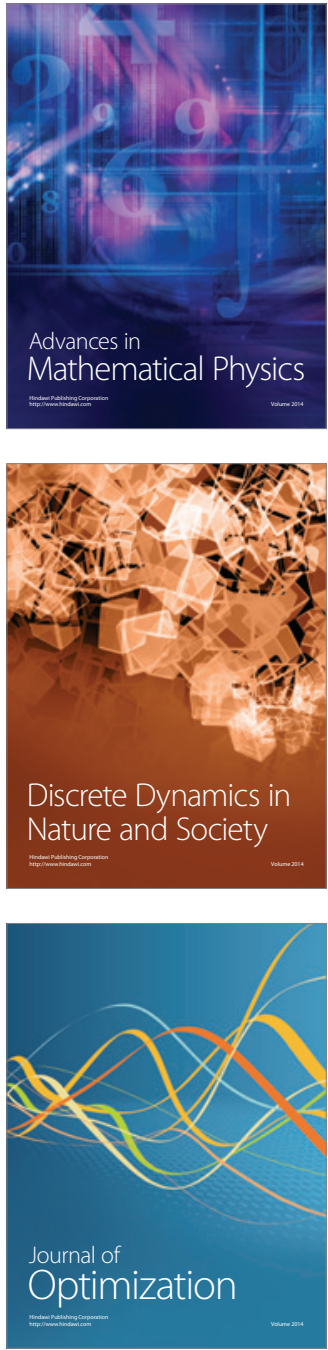